\title{
ELUCIDATION THE TOXICITY MECHANISM OF ZINC OXIDE NANOPARTICLE USING MOLECULAR DOCKING APPROACH WITH PROTEINS
}

\author{
KRISHNA PAL SINGH ${ }^{1}$, ANUPAM DHASMANA ${ }^{2}$, QAMAR RAHMAN ${ }^{1}$ \\ ${ }^{1}$ Department of Amity Institute of Biotechnology, Amity University, Lucknow, Uttar Pradesh, India. ${ }^{2}$ Department of Himalayan, School of \\ Biosciences, Swami Rama Himalayan University, Dehradun, Uttarakhand, India. Email: krishnapalsingh22@gmail.com
}

Received: 01 November 2017, Revised and Accepted: 04 December 2017

\begin{abstract}
Objective: At present, toxicological tests are resource-intensive, time-consuming and require a large pool of animal models for toxicity assessment. To speed up the toxicity evaluation and to reduce animal suffering during toxicity assessment, the use of alternative methods including computational models is in high demand. The computational toxicity prediction methods are very helpful for the regulatory bodies to quickly assess the health impact of nanomaterial materials. In the present work, we have examined the mechanism of zinc oxide nanoparticle (ZnO-NP)-proteins interaction and their effect of surface chemistries of $\mathrm{ZnO}-\mathrm{NP}$ on the bioactive conformation of chemokines and other cytological proteins using in silico molecular docking approaches.
\end{abstract}

Methods: Molecular docking study was conducted using AutoDock 4.0 version and the visualization result using Discover Studio 4.0.

Results: In the present study, we observed that ZnO-NP has high binding affinity with the mitogen-activated protein kinases (P-38), nuclear factor kappa-light-chain-enhancer of activated B cell (NF-kB) proteins, and matrix metallopeptidase-9 with docking energies -8.81, -7.64 , and $-7.27 \mathrm{Kcal} / \mathrm{Mol}$, respectively, involving with hydrogen, metal acceptor, and electrostatic interaction. The top interacting amino acid residues with ZnO-NP are GLY, PHE, ARG, ASP, GLN, and ASN.

Conclusion: Thus, based on the molecular docking studies, we determine that the ZnO-NP is strongly interacting with the chemokines and other cytological proteins thus responsible for blocking of the activation stimuli for these proteins to initiate the biological signals for the proper functioning. We have also extracted the information of interaction pattern of ZnO-NP with the surface-enriched amino acid residues of chemokine and cytological proteins using molecular docking approach.

Keywords: Zinc oxide nanoparticle, Molecular docking, Nanoparticle-protein interaction, Toxicity.

(c) 2018 The Authors. Published by Innovare Academic Sciences Pvt Ltd. This is an open access article under the CC BY license (http://creativecommons. org/licenses/by/4. 0/) DOI: http://dx.doi.org/10.22159/ajpcr.2018.v11i3.23384

\section{INTRODUCTION}

Nanomaterials are of great interest because of their novel properties, including a large specific surface area and high reaction activity [1,2]. In current scenario, expansion in nanotechnology engineering has increased the prompt development of many applications for nanomaterials such as metal nanoparticles (NP) (e.g., gold and silver), metal oxide NP (e.g., CuO, TiO2, and zinc oxide NP [ZnO]), C60 fullerenes nanocrystals, and carbon nanotubes (CNTs) [3-6]. In case of NP, the diverse materials were found, for example, gold, silica, titanium, CNTs, zinc, and quantum dots have shown unique mechanisms of proteins modifications, lipid peroxidation, and DNA fragmentation reactive oxygen species possible that lead to cellular damage and other several disorders including cancer [7]. $\mathrm{ZnO}$ is most widely commonly utilized as a group of nanomaterials [8]. The progressive utilized ZnO-NPs in sunscreens, biosensors, food additives, pigments, rubber manufacture, and electronic materials. Rise concerns have also been its unintentional health, environmental impacts, and negative effects on the survival and growth of organisms [9]. A number of in vitro studies proved that ZnO-NPs are toxic for mammalian cells and are even more toxic than other nanoscale structures of metallic oxide $[10,11]$. The interaction of nanomaterial with proteins results in physiological changes that lead to various pathological phenotypes. However, the mechanism underlying these changes remain poorly understood. In this study, we have selected some of the chemokine and cytological proteins which were previously suggested to interact with $\mathrm{ZnO}$-NP in various experimental settings [12-16]. It was experimentally verified that the interaction of ZnO-NP with ICAM-1, IL8, IL1B, P-38, and nuclear factor (NF-kB) induces expression of these proteins $[12,17-19]$, similarly in case of
CCL18 and CD35, interaction resulted in decreased expression [14,20]. The matrix metallopeptidase-9 (MMP-9) interacts with ZnO-NP which results in the increase enzymatic activity [14]. ZnO-NPs get absorbed systemically in the liver, adipose, and pancreas, which can elevate the level of zinc in the body [21]. The stimulation of oxidative stress is the vital part of the cytotoxicity of ZnO-NPs [22]. The major challenge in the current study is to deal with the size, density, and surface property variation of the nanomaterial because in the case of in vitro and in vivo experimental settings, these parameters were either poorly determined or with very broad range [23]. Furthermore, very few methods and studies are available where the toxicity of $\mathrm{ZnO}$-NP was assessed using a computational method. In the case of new materials, computational toxicity prediction methods are now frequently used by regulatory bodies to quickly assess the health hazards. In the present study, we have investigated the mechanism of $\mathrm{ZnO}-\mathrm{NP}$-protein interaction and their effect of surface chemistries of $\mathrm{ZnO}-\mathrm{NP}$ on the bioactive conformation of chemokines and other cytological proteins using in silico molecular docking approaches. We have selected those proteins which have already reported interaction with $\mathrm{ZnO}-\mathrm{NP}$ and also well-established in the earlier works using in vitro techniques, and the in -silico results have been discussed comparatively. We have specifically investigated the effect of NP toxicity and an insight into the regularity of interaction has been attempted between NPs with specific amino acids.

\section{METHODS}

Retrieval of chemokine and other cytological proteins threedimensional (3D) conformation

The coordinates of selected proteins (Table 1) were obtained from the RCSB protein data bank (http://www.rcsb.org/pdb/home/ 
home.do). The protein molecules were prepared for the molecular interaction studies using the prepare protein protocols of Biovia Discovery Studio 4.0 software suit. Prepared protein protocol fixed various protein structure errors such as missing atoms in incomplete residues, missing loop regions, alternate conformations (disorder), non-standard atom names, and incorrect protonation state of titratable residues. For molecular docking studies, we defined the active sites of proteins using in silco literature, [24] and rest of active site of proteins were retrieved using $\mathrm{COACH}$ server [25]. $\mathrm{COACH}$ is a meta-server for the prediction of protein-ligand binding site prediction. The prediction of the active site is mainly based on two comparative methods, TM-SITE and S-SITE, which recognize ligandbinding templates from the BioLiP protein function database [25] by binding-specific substructure and sequence profile comparisons. The assessment of the active site was done using the confidence score (C-score) that determined the accuracy of the active site prediction. C-score ranges $0-1$, where a higher score indicates a more reliable prediction and this score is defined based on the quality of the threading alignments and the convergence of the I-TASSER's structural assembly refinement simulations.

$$
\mathrm{C} \text {-score }=\ln \left[\frac{\mathrm{M}}{\mathrm{M}_{\mathrm{tot}}} \times \frac{1}{<\mathrm{RMSD}>} \times \frac{1}{7} \sum_{\mathrm{i}=1}^{7} \frac{\mathrm{Z}(\mathrm{i})}{\mathrm{Z}_{0}(\mathrm{i})}\right]
$$

Where $M$ is the number of structure decoys in the cluster and $M_{\text {tot }}$ is the total number of decoys generated during the I-TASSER simulations, RMSD is the average deviation of the decoys from the cluster centroid. $\mathrm{Z}(\mathrm{i})$ is the $\mathrm{Z}$-score of the best template generated by $\mathrm{i}^{\text {th }}$ threading in the seven LOMETS programs and $\mathrm{Z}_{0}(\mathrm{i})$ is a program-specified Z-score cutoff for distinguishing between good and bad templates. The actives site residues of selected chemokine and other cytological proteins are shown in Fig. 1.

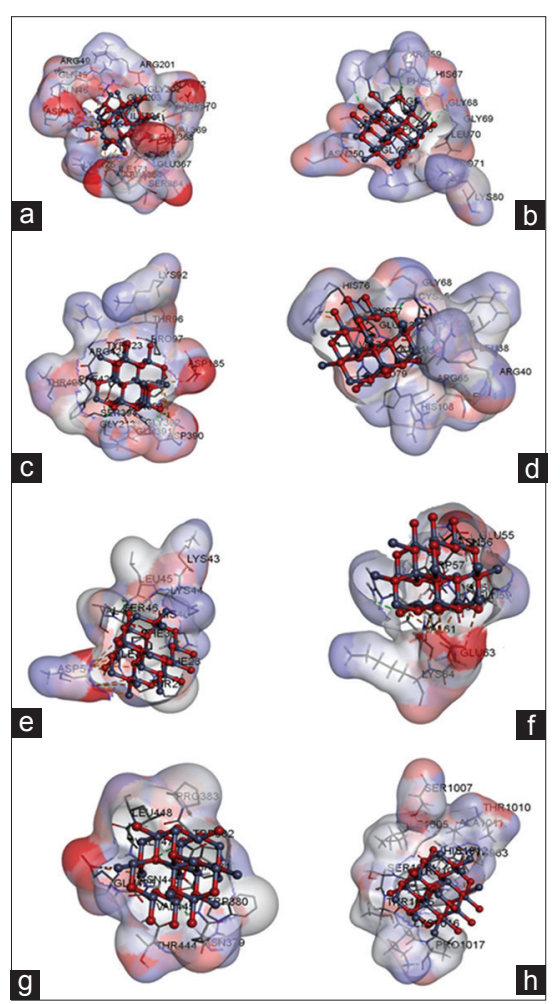

Fig. 1: Interaction of zinc oxide nanoparticle with the activation site cavity of Chemokines and other cytological proteins. (a) p-38 protein, (b) nuclear factor-kB protein, (c) matrix metallopeptidase-9 protein, (d) IL-1B protein, (e) CCL-18 protein, (f) IL8 protein, (g) ICAM-1 protein, (h) CD-35 protein, respectively

\section{D model of ZnO-NP}

3D nano-system of ZnO-NP with a dimension of $1 \mathrm{~nm}$ was built with the help of build protocol of Biovia Material Studio 7.0. The NP was optimized using Forcite geometry optimization protocol [26]. The geometry optimization was done using COMPASS force field at 1NVT, $300 \mathrm{~K}$ for $100 \mathrm{ps}$.

\section{Molecular docking of ZnO-NP with proteins}

Molecular docking plays important part in the study the interaction of ligand with active site residues of the receptor [27]. Molecular docking of $\mathrm{ZnO}$-NP with the chemokines and other cytological proteins were performed using the AutoDock4.0 [28,29]. AutoDock has been already implemented by several researchers to study numerous proteins-NP interactions [30-32]. Before going for molecular docking, hydrogen atoms were added to all target proteins. Kollman united charges and salvation parameters were added to the proteins. Gasteiger charge was added to the ZnO-NP. Grid box was set in such a manner that it will cover the active sites of chemokines and cytological proteins so that the interaction will take place on the active site of proteins. The values were set to $60 \times 60 \times 603 \AA$ in $X, Y$, and Z-axis of the grid point. The grid point spacing was kept at $0.375 \AA$. Lamarckian Genetic Algorithm [33] and flexible docking calculations were used for the docking purpose. $\mathrm{ZnO}$-NP was placed at random starting position random orientation and torsions were used. For the translation, quaternion and torsion steps were consider as default values in AutoDock. In AutoDock standard docking protocol for ligand docking consisted of 10 independent runs per ligand, considering an initial population of 50 randomly placed individuals, with $2.5 \times 106$ energy evaluations, a maximum number of 27,000 iterations, a mutation rate of 0.02 , a crossover rate of 0.80 , and an elitism value of 1 . The local search for an individual in the population was 0.06 , using a maximum of 300 iterations per local search. After docking, the 10 solutions were clustered into groups with RMS deviations lower than $1.0 \AA$. The clusters were ranked by the lowest energy representative of each cluster. Discovery Studio 4.0 was used to analyze the resultant conformations for the intermolecular interactions and binding energy.

Investigate the consequence of the interaction of $\mathrm{ZnO}-\mathrm{NP}$ on biological processes

After molecular docking study, it is important to understand the biological processes in which these proteins were plays important role. We can comprehensive understand and actual impact of the docking in the biological system. To understand the biological impact, we have retrieved all the biological function of the proteins which were taken in the study in a form of network that interacts to accomplish a process to the level with in the cell or organism. Different biological functions of chemokines and other cytological proteins were accessed using PANTHER (http://pantherdb.org) (protein analysis through evolutionary relationships). Classification system was designed to classify proteins (and their genes) to facilitate high-throughput analysis.

\section{RESULTS AND DISCUSSION}

In this study, we have taken cellular proteins into consideration for the examination of their interactions with $\mathrm{ZnO} \mathrm{O}$ - $\mathrm{N}$ and the possible functions that can affect the physiology. All the putative targets of ZnO-NP and the active site predictions were carried out using COACH meta-server. The best active sites prediction base on the $\mathrm{C}$-score of $\mathrm{COACH}$ meta-server for IL-1B and CCL-18 proteins are 0.23 and 0.26 . In our docking studies, we have defined the active site of proteins to perform control docking with ZnO-NP. The best docking poses of ZnO-NP with proteins based on AutoDock binding energy were analyzed further. The best docking energy of ZnO-NP with P-38 (-8.81 Kcal/Mol), a total of 4 hydrogen bonds with GLY202, GLU203, ILE204, VAL369 and 4 electrostatic bonds formed with APS43, GLU203, ASP370, PHE371, and also 2 Metal Acceptor bonds with ASP365 and VAL369 amino acid residues which clearly indicate that the ZnO-NP strongly bind to the active site cavity. We observed the secondbest docking energy $(-7.64 \mathrm{Kcal} / \mathrm{Mol})$ of $\mathrm{ZnO}-\mathrm{NP}$ with $\mathrm{NF}-\mathrm{kB}$ protein, it forms 5 hydrogen bonds with ARG57, HIS67, GLY68, PR071, 




Fig. 2: Illustration of the interaction network of zinc oxide nanoparticle (ZnO-NP) with chemokines and other cytological proteins. Nodes with different colors represent various Biological functions of proteins which were inhibited by ZnO-NP. The figure legend represents the function of these proteins

Table 1: Docking energies and bond information of ZnO-NP with chemokines and other cytological proteins

\begin{tabular}{|c|c|c|c|c|c|c|}
\hline \multirow[t]{2}{*}{ Proteins } & \multirow[t]{2}{*}{ NP } & \multirow[t]{2}{*}{ Energy in (Kcal/Mol) } & \multirow[t]{2}{*}{ Total number of $\mathrm{H}$ Bond } & \multirow[t]{2}{*}{ Metal acceptor } & \multicolumn{2}{|c|}{ Electrostatic } \\
\hline & & & & & Pi-Cation & Attractive charge \\
\hline 1I1B (IL-1B) & ZnO-NP & -3.23 & 3 & - & - & 3 \\
\hline $1 \mathrm{GKG}(\mathrm{CD} 35)$ & & -3.92 & 2 & 1 & 1 & - \\
\hline 1L6J (MMP-9) & & -7.27 & 5 & 2 & 1 & 1 \\
\hline 1IL8 (IL8) & & -5.14 & 2 & 2 & - & 1 \\
\hline 4MHE (CCL-18) & & -3.13 & 3 & 4 & - & - \\
\hline 1P53 (ICAM-1) & & -4.95 & 2 & 1 & - & 2 \\
\hline 1SVC (NF-kB) & & -7.64 & 5 & 3 & 1 & - \\
\hline $3 W 8 Q(P-38)$ & & -8.81 & 4 & 2 & 1 & 3 \\
\hline
\end{tabular}

and SER243, 3 metal acceptor bonds with GLY55, ARG57, and HIS67 and 1 electrostatic bond with PHE56 amino acid residues, and it also has strong binding in the active site cavity. On the other side, we observed least binding affinity for ZnO-NP with CCL-18 (-3.13 Kcal/Mol), whereas it forms only 3 hydrogen bonds and 4 metal acceptors with GLY5, ASN7, and GLN34 amino acid residues. The binding energies of other proteins, namely, ICAM-1, IL8, MMP-9, IL-1B, and CD-35 proteins with ZnO-NP are listed in Table 1.

From the molecular docking interactions, we have extracted the key residues of proteins which play important role in the binding of $\mathrm{ZnO}$ NP more efficiently GLY which is hydrophobic in nature and present in $1 \mathrm{SVC}, 3 \mathrm{~W} 8 \mathrm{Q}, 1 \mathrm{I} 6 \mathrm{~J}$, and $1 \mathrm{I} 1 \mathrm{~B}$ proteins. The rest of key residues interacting with proteins are PHE, ARG, ASP, GLN, and ASN and their nature were given in Table 2 .

The amino acids show least interaction with ZnO-NP is LYS, VAL, THR, TYR, TRP, and LEU. Details of molecular interactions of $\mathrm{ZnO}$ NP with chemokines and other cytological proteins are provided in Supplementary Table 1 and the best interacting pose was shown in Fig. 1. The molecular docked proteins have a specific role in biological processes which can affect by the interaction with $\mathrm{ZnO}$ NP. The outcome from this study clearly indicated that the ZnO-NP inhibits the process of response to a stimulus, cellular process, and biological adhesion more effectively and other processes were given in Fig. 2.
Fig. 2: Illustration of the interaction network of zinc oxide nanoparticle (ZnO-NP) with chemokines and other cytological proteins. Nodes with different colors represent various Biological functions of proteins which were inhibited by $\mathrm{ZnO}$-NP. The figure legend represents the function of these proteins

Protein ICAM-1 also known as CD54 (cluster of differentiation 54) has an important role in cell-cell signaling for stabilization of cell-cell interaction and facilitates the leukocyte endothelial transmigration [34,35]. The ICAM-1 plays a significant role in spermatogenesis due to its antagonistic effect on the tight junctions forming the blood test is barrier [34]. P-38 has an important function in cell-cell signaling against stress and is responsive to stress stimuli, such as cytokines, ultraviolet irradiation, heat shock, and osmotic shock. It is also involved in cell differentiation, apoptosis, and autophagy $[36,37]$. Similarly, NF-kB plays a very important role in several cellular functions. NF-kB controls the cellular responses as it belongs to the category of "rapid-acting" primary transcription factor, i.e., a transcription factor which was present in cells in an inactive state and does not undergo new protein synthesis to become activated [38,39]. Several innate and adaptive immune response genes are regulated by a transcription factor (NF-kB) [40-42]. Interaction of $\mathrm{ZnO}-\mathrm{NP}$ with proteins might be altering the functional properties, as results may affect stabilization of cell-cell interactions. It is a newer approach to determine the $\mathrm{ZnO}-\mathrm{NP}$ interaction with proteins using docking, without any surface modification of NP. 
Table 2: Complete H-bond interaction analysis of amino acid residues of proteins with ZnO-NP

\begin{tabular}{|c|c|c|c|c|c|c|c|c|c|c|c|}
\hline $\begin{array}{l}\text { Amino } \\
\text { acids } \\
\text { residues }\end{array}$ & 1SVC & $3 W 8 Q$ & 1I6J & 1 IL8 & 1GKG & $1 \mathrm{P53}$ & 1I1B & 4MHE & $\begin{array}{l}\text { Charged (side } \\
\text { chains often } \\
\text { make salt } \\
\text { bridges) }\end{array}$ & $\begin{array}{l}\text { Polar (usually } \\
\text { participate in } \\
\text { hydrogen bonds } \\
\text { as proton donors } \\
\text { or acceptors) }\end{array}$ & $\begin{array}{l}\text { Hydrophobic (normally } \\
\text { buried inside the } \\
\text { protein core) }\end{array}$ \\
\hline PHE & $\checkmark$ & $\checkmark$ & $\checkmark$ & - & - & - & - & - & - & - & $\checkmark$ \\
\hline ARG & $\checkmark$ & - & $\checkmark$ & $\checkmark$ & - & - & - & - & $\checkmark$ & - & - \\
\hline GLY & $\checkmark$ & $\checkmark$ & $\checkmark$ & - & - & - & - & $\checkmark$ & - & - & $\checkmark$ \\
\hline PRO & $\checkmark$ & - & - & - & $\checkmark$ & - & - & - & - & - & $\checkmark$ \\
\hline SER & $\checkmark$ & - & - & - & $\checkmark$ & - & - & - & - & $\checkmark$ & - \\
\hline GLN & - & - & $\checkmark$ & $\checkmark$ & - & - & - & $\checkmark$ & - & $\checkmark$ & - \\
\hline LYS & - & - & - & - & - & - & $\checkmark$ & - & $\checkmark$ & - & - \\
\hline ILE & - & $\checkmark$ & - & - & - & - & - & - & - & - & $\checkmark$ \\
\hline VAL & - & $\checkmark$ & - & - & - & - & - & - & - & - & $\checkmark$ \\
\hline ASP & - & $\checkmark$ & $\checkmark$ & - & - & - & $\checkmark$ & - & $\checkmark$ & - & - \\
\hline GLU & - & $\checkmark$ & - & $\checkmark$ & - & $\checkmark$ & - & - & $\checkmark$ & - & - \\
\hline ASN & - & - & - & $\checkmark$ & - & $\checkmark$ & - & $\checkmark$ & - & $\checkmark$ & - \\
\hline TYR & - & - & $\checkmark$ & - & - & - & - & - & - & $\checkmark$ & - \\
\hline TRP & - & - & - & - & - & $\checkmark$ & - & - & - & $\checkmark$ & - \\
\hline LEU & - & - & - & - & - & - & $\checkmark$ & - & - & - & - \\
\hline
\end{tabular}

The $\checkmark$ sign indicates that the particular H-bond is present while - indicates its absence and also the nature of interacting amino acid residues, ZnO-NP: Zinc oxide nanoparticle

\section{CONCLUSION}

Using molecular docking as computational approaches could be adopted for the screening of all the NPs, which can bind to the target with experimental or modeled structures. A number of studies already reported that engineer NP particles (ENPs) interact with the biological macromolecules. Recent studies have shown that ENPs inhibit enzyme activity due to their interaction with the active site or binding directly with the substrate [43,44]. Using molecular docking studies, we conclude that the $\mathrm{ZnO}-\mathrm{NP}$ is strongly interacting with the chemokines and other cytological proteins on its activation site and thus responsible for blocking of the activation stimuli for chemokines, and other cytological proteins to initiate the biological signals for the proper functioning of these proteins. In contrast, from the above results, it indicates the key interacting amino acid residues with $\mathrm{ZnO}$ $\mathrm{NP}$ and their nature. Which plays important role in the interaction of ZnO-NP with proteins.

\section{ACKNOWLEDGMENTS}

We express our gratitude to Amity University Uttar Pradesh, Lucknow Campus, for providing the laboratory facilities and encouragement.

\section{AUTHORS CONTRIBUTIONS}

Mr. Krishna Pal Singh: Carried out the Research work. Dr. Qamar Rahman: Provided guidance, critical review and revision. Dr. Anupam Dhasmana: Helped while caring the experiments.

\section{CONFLICT OF INTERESTS}

The authors declare that they have no conflict of interest.

\section{REFERENCES}

1. Amelia M, Lincheneau C, Silvi S, Credi A. Electrochemical properties of cdSe and cdTe quantum dots. Chem Soc Rev 2012;41:5728-43.

2. Yan L, Zheng YB, Zhao F, Li S, Gao X, Xu B, et al. Chemistry and physics of a single atomic layer: Strategies and challenges for functionalization of graphene and graphene-based materials. Chem Soc Rev 2012:41:97-114.

3. Nel A, Xia T, Mädler L, Li N. Toxic potential of materials at the nanolevel. Science 2006;311:622-7.

4. Buzea C, Pacheco II, Robbie K. Nanomaterials and nanoparticles: Sources and toxicity. Biointerphases 2007;2:MR17-71.
5. De Stefano D, Carnuccio R, Maiuri MC. Nanomaterials toxicity and cell death modalities. J Drug Deliv 2012;2012:167896.

6. Oberdörster G. Nanotoxicology: In vitro-in vivo dosimetry. Environ Health Perspect 2012;120:A13.

7. Oberdörster G, Oberdörster E, Oberdörster J. Nanotoxicology: An emerging discipline evolving from studies of ultrafine particles. Environ Health Perspect 2005;113:823-39.

8. Fan Z, Lu JG. Zinc oxide nanostructures: Synthesis and properties. J Nanosci Nanotechnol 2005;5:1561-73.

9. Nations S, Wages M, Cañas JE, Maul J, Theodorakis C, Cobb GP, et al. Acute effects of $\mathrm{Fe} 2 \mathrm{O} 3, \mathrm{TiO} 2, \mathrm{ZnO}$ and $\mathrm{CuO}$ nanomaterials on Xenopus laevis. Chemosphere 2011;83:1053-61.

10. Jeng HA, Swanson J. Toxicity of metal oxide nanoparticles in mammalian cells. J Environ Sci Heal Part A 2006;41:2699-711.

11. Horie M, Nishio K, Fujita K, Endoh S, Miyauchi A, Saito Y, et al. Protein adsorption of ultrafine metal oxide and its influence on cytotoxicity toward cultured cells. Chem Res Toxicol 2009;22:543-53.

12. Wu W, Samet JM, Peden DB, Bromberg PA. Phosphorylation of p65 is required for zinc oxide nanoparticle-induced interleukin 8 EXPRESSION in human bronchial epithelial cells. Environ Health Perspect 2010;118:982-7.

13. Hackenberg S, Scherzed A, Technau A, Kessler M, Froelich K, Ginzkey C, et al. Cytotoxic, genotoxic and pro-inflammatory effects of zinc oxide nanoparticles in human nasal mucosa cells in vitro. Toxicol In Vitro 2011;25:657-63.

14. Babin K, Antoine F, Goncalves DM, Girard D. $\mathrm{TiO} 2, \mathrm{ceO} 2$ and znO nanoparticles and modulation of the degranulation process in human neutrophils. Toxicol Lett 2013;221:57-63.

15. Yuan L, Wang Y, Wang J, Xiao H, Liu X. Additive effect of zinc oxide nanoparticles and isoorientin on apoptosis in human hepatoma cell line. Toxicol Lett 2014;225:294-304.

16. Suzuki Y, Tada-Oikawa S, Ichihara G, Yabata M, Izuoka K, Suzuki $\mathrm{M}$, et al. Zinc oxide nanoparticles induce migration and adhesion of monocytes to endothelial cells and accelerate foam cell formation. Toxicol Appl Pharmacol 2014;278:16-25.

17. Li CH, Liao PL, Shyu MK, Liu CW, Kao CC, Huang SH, et al. Zinc oxide nanoparticles-induced intercellular adhesion molecule 1 expression requires $\mathrm{Rac} 1 / \mathrm{Cdc} 42$, mixed lineage kinase 3, and c-Jun N-terminal kinase activation in endothelial cells. Toxicol Sci 2012;126:162-72.

18. Sharma V, Anderson D, Dhawan A. Zinc oxide nanoparticles induce oxidative DNA damage and ROS-triggered mitochondria mediated apoptosis in human liver cells (HepG2). Apoptosis 2012;17:852-70.

19. Song J, Du L, Feng Y, Wu W, Yan Z. Pyroptosis induced by zinc oxide nanoparticles in A549 cells. Wei Sheng Yan Jiu 2013;42:273-6.

20. Chuang KJ, Lee KY, Pan CH, Lai CH, Lin LY, Ho SC, et al. Effects of zinc oxide nanoparticles on human coronary artery endothelial cells. Food Chem Toxicol 2016;93:138-44. 
21. Umrani RD, Paknikar KM. Zinc oxide nanoparticles show antidiabetic activity in streptozotocin-induced type 1 and 2 diabetic rats. Nanomedicine (Lond) 2014;9:89-104.

22. Pandurangan M, Veerappan M, Kim DH. Cytotoxicity of zinc oxide nanoparticles on antioxidant enzyme activities and mRNA expression in the cocultured $\mathrm{C} 2 \mathrm{C} 12$ and 3T3-L1 cells. Appl Biochem Biotechnol 2015; $175: 1270-80$

23. Kumar A, Dhawan A, Shanker R. The need for novel approaches in ecotoxicity of engineered nanomaterials. J Biomed Nanotechnol 2011;7:79-80

24. Ranjan S, Dasgupta N, Chinnappan S, Ramalingam C, Kumar A. A novel approach to evaluate titanium dioxide nanoparticle-protein interaction through docking: An insight into mechanism of action. Proc Natl Acad Sci India Sect B Biol Sci 2015;87:937-43.

25. Yang J, Roy A, Zhang Y. BioLiP: A semi-manually curated database for biologically relevant ligand-protein interactions. Nucleic Acids Res 2013;41:D1096-103.

26. Rahman S, Farooqui SA, Rai A, Kumar R, Santra C, Prabhakaran VC, et al. Mesoporous TUD-1 supported indium oxide nanoparticles for epoxidation of styrene using molecular O2. RSC Adv 2015;5:46850-60.

27. Sahare $\mathrm{P}, \mathrm{Moon} \mathrm{A}$. In silico modelling of $\beta$-lactam resistant enterococcus faecalis pbp4 and its interactions with various phyto-ligands. Int J Pharm Pharm Sci 2016;8:151-5.

28. Dharani R, Ranjitha R, Sripathi R, Ali Muhammad KS, Ravi S. Docking studies in target proteins involved in antibacterial action mechanisms: Alkaloids isolated from Scutellaria genus. Asian J Pharm Clin Res 2016;9:121-5

29. Morris GM, Goodsell DS, Huey R, Olson AJ. Distributed automated docking of flexible ligands to proteins: Parallel applications of autoDock 2.4. J Comput Aided Mol Des 1996;10:293-304.

30. Benyamini H, Shulman-Peleg A, Wolfson HJ, Belgorodsky B, Fadeev L, Gozin M. Interaction of C60-Fullerene and carboxyfullerene with proteins: Docking and binding site alignment. Bioconjug Chem 2006; 17:378-86.

31. Baweja L, Gurbani D, Shanker R, Pandey AK, Subramanian V, Dhawan A, et al. C60-fullerene binds with the ATP binding domain of human DNA topoiosmerase II alpha. J Biomed Nanotechnol $2011 ; 7: 177-8$
32. Patel A, Smita S, Rahman Q, Gupta SK, Verma MK. Single wall carbon nanotubes block ion passage in mechano-sensitive ion channels by interacting with extracellular domain. J Biomed Nanotechnol 2011;7:183-5.

33. Goodsell DS, Morris GM, Olson AJ. Automated docking of flexible ligands: Applications of auto dock. J Mol Recognit 1996;9:1-5.

34. Xiao X, Mruk DD, Cheng CY. Intercellular adhesion molecules (ICAMs) and spermatogenesis. Hum Reprod Update 2013;19:167-86.

35. Bai R, Yi S, Zhang X, Liu H, Fang X. Role of ICAM-1 polymorphisms (G241R, K469E) in mediating its single-molecule binding ability: Atomic force microscopy measurements on living cells. Biochem Biophys Res Commun 2014;448:372-8.

36. Kao YT, Hsu WC, Hu HT, Hsu SH, Lin CS, Chiu CC, et al. Involvement of p38 mitogen-activated protein kinase in acquired gemcitabineresistant human urothelial carcinoma sublines. Kaohsiung J Med Sci 2014:30:323-30.

37. Javadov S, Jang S, Agostini B. Crosstalk between mitogen-activated protein kinases and mitochondria in cardiac diseases: Therapeutic perspectives. Pharmacol Ther 2014;144:202-25.

38. Pavlová S, Klucska K, Vašíček D, Ryban L, Harrath AH, Alwasel SH, et al. The involvement of SIRT1 and transcription factor NF-кB (p50/ p65) in regulation of porcine ovarian cell function. Anim Reprod Sci 2013;140:180-8

39. Marinho HS, Real C, Cyrne L, Soares H, Antunes F. Hydrogen peroxide sensing, signaling and regulation of transcription factors. Redox Biol 2014;2:535-62.

40. Colvin VL. The potential environmental impact of engineered nanomaterials. Nat Biotechnol 2003;21:1166-70.

41. Hayden MS, West AP, Ghosh S. NF- $\kappa B$ and the immune response. Oncogene 2006;25:6758-6780.

42. Jin J, Hu H, Li HS, Yu J, Xiao Y, Brittain GC, et al. Noncanonical NF$\kappa \mathrm{B}$ pathway controls the production of Type I interferons in antiviral innate immunity. Immunity 2014;40:342-54.

43. Kain J, Karlsson HL, Möller L. DNA damage induced by micro-and nanoparticles - interaction with FPG influences the detection of DNA oxidation in the comet assay. Mutagenesis 2012;27:491-500.

44. Magdolenova Z, Collins A, Kumar A, Dhawan A, Stone V, Dusinska M, et al. Mechanisms of genotoxicity. A review of in vitro and in vivo studies with engineered nanoparticles. Nanotoxicology 2014;8:233-78. 
Supplementary Table 1: Detail interaction of ZnO-NP with chemokines and other cytological proteins

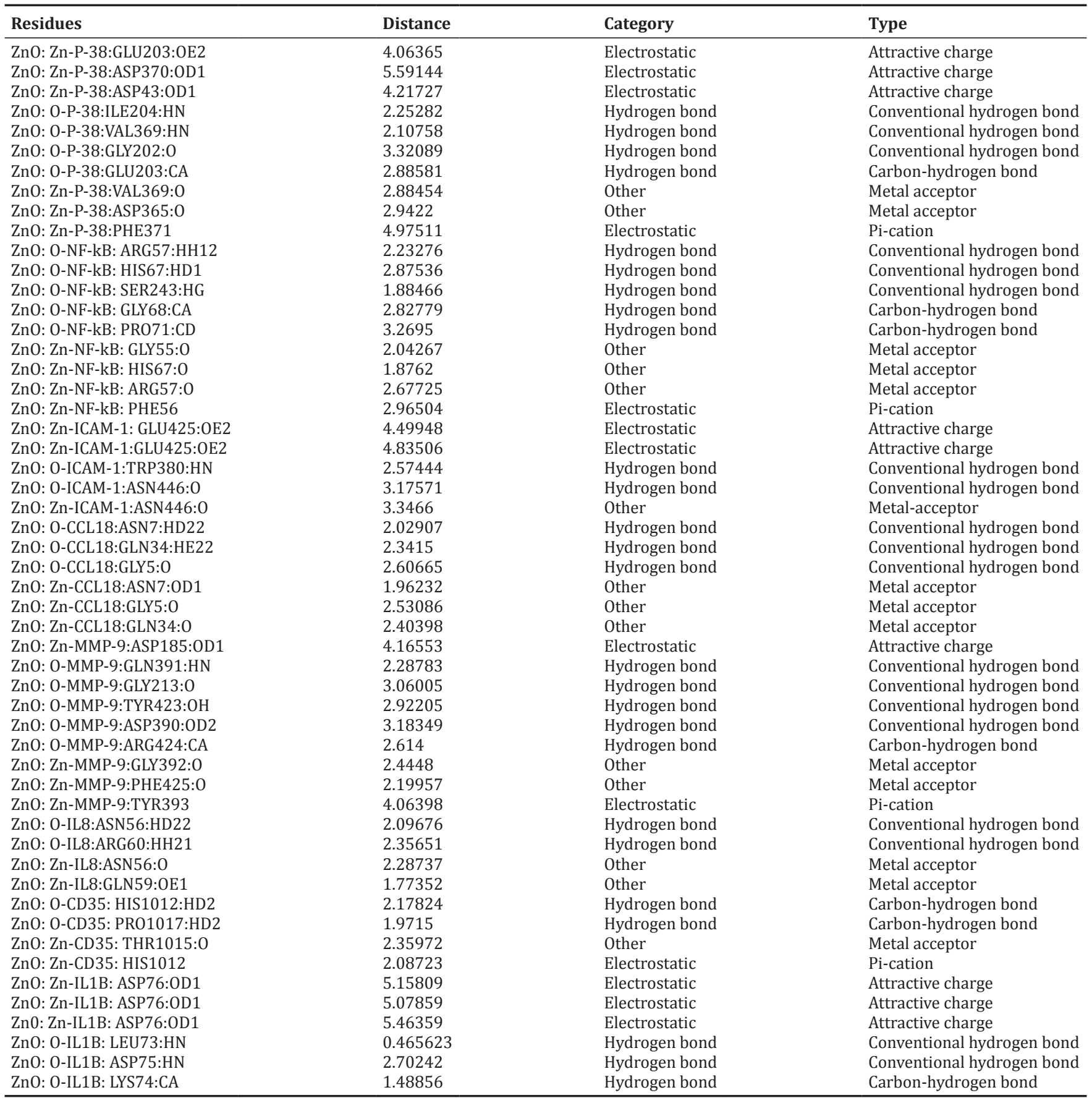

